\title{
Controlling Below-Threshold Nonsequential Double Ionization via Quantum Interference
}

\author{
A. S. Maxwell ${ }^{*}$ and C. Figueira de Morisson Faria ${ }^{\dagger}$ \\ Department of Physics and Astronomy, University College London, Gower Street, London WC1E 6BT, United Kingdom
}

(Received 20 July 2015; revised manuscript received 14 January 2016; published 4 April 2016)

\begin{abstract}
We show through simulation that quantum interference in nonsequential double ionization can be used to control the recollision excitation with subsequent ionization (RESI) mechanism. This includes the shape, localization, and symmetry of RESI electron-momentum distributions, which may be shifted from a correlated to an anticorrelated distribution or vice versa, far below the direct ionization threshold intensity. As a testing ground, we reproduce recent experimental results by employing specific coherent superpositions of excitation channels. We examine two types of interference, from electron indistinguishability and intracycle events, and from different excitation channels. These effects survive focal averaging and transverse-momentum integration.
\end{abstract}

DOI: 10.1103/PhysRevLett.116.143001

Correlation and anticorrelation have been extensively studied in strong-field, laser-induced nonsequential double ionization (NSDI). Particularly with the Cold Target Recoil Ion Momentum Spectroscopy technique, information about the electron momenta has become experimentally accessible since the early 2000s. Several features in these distributions provide information about the type of interaction by which NSDI occurs, and the physical mechanisms behind it. It is commonly accepted that NSDI results from the laserinduced inelastic recollision of an electron with its parent ion [1]. If the driving-field intensity is high enough, upon recollision the first active electron releases a second electron by electron-impact (EI) ionization. In contrast, for the socalled below-threshold intensities, the kinetic energy transferred from the first electron to the core is not sufficient to free the second electron. Instead, the recolliding electron imparts only enough energy that the second electron is excited and then ionized with a time delay. This mechanism is known as recollision excitation with subsequent ionization (RESI).

Over the years, the prevalent view has been that, in EI, the two electrons will exhibit correlated momenta as a consequence of their being released simultaneously. In contrast, for RESI, back-to-back emission will occur due to the time delay between recollision of the first electron and ionization of the second electron. This view has been backed using classical models, which have reproduced many of the key features encountered in experiments (for reviews, see Ref. [2]). Such models also exhibit excellent agreement with the outcome of other methods, such as the full solution of the time-dependent Schrödinger equation [3] and the strong-field approximation (SFA) [4-6]. The abovementioned studies, performed for EI, suggested that quantum mechanical features such as interference will not survive integration over momentum components perpendicular to the laser-field polarization and focal averaging, which is the typical scenario in NSDI experiments. These conclusions were then extrapolated to RESI without much evidence.
Classical models have also successfully reproduced the anticorrelated behavior observed in early RESI experiments [7-10]. However, recent RESI experiments have found that electron-momentum distributions may occ+ur in a variety of shapes, from cross-shaped distributions localized along the axis [11] to probability densities spread between all four quadrants of the plane spanned by the parallel electronmomentum components $[12,13]$. These findings contradict the simple explanation that a time delay will lead to anticorrelated electron momenta. A wealth of shapes have also been observed in many theoretical studies, ones as diverse as the SFA and similar methods [14-16] to classicaltrajectory computations [17-19]. If quantum interference is absent, the SFA retains fourfold symmetry for RESI distributions, in agreement with rigorous momentum constraints [15]. Recently, however, SFA computations have shown that this symmetry can be broken if the interference stemming from different excitation channels is incorporated [20]. Therein, it has been argued that interchannel interference is paramount for obtaining anticorrelated RESI distributions. However, no attention has been paid to symmetryrelated interference, and no explicit conditions have been provided for either. Another open question is whether, given a RESI distribution of a particular shape, the intermediate state of the second electron can be reconstructed.

In this Letter, we show that one may obtain correlated, anticorrelated, cross-, or ring-shaped RESI distributions in a SFA computation by choosing appropriate coherent superpositions of excitation channels. We reproduce recent experimental results in which dramatic variations in the shapes of RESI distributions have been observed [12]. The features encountered are related to the interplay between two types of interference, involving (a) events which are displaced by half a cycle and those present due to the symmetry of indistinguishable electrons, and (b) different channels of excitation for the second electron. These effects survive transverse-momentum integration and focal averaging, individually break the fourfold symmetry of the RESI 
distributions, and may be used to manipulate electronelectron correlation.

In the SFA and in atomic units, the RESI transition amplitude related to the $c$ th excitation channel reads

$$
\begin{aligned}
& M^{(c)}\left(\mathbf{p}_{1}, \mathbf{p}_{2}\right) \\
& =\int_{-\infty}^{\infty} d t \int_{-\infty}^{t} d t^{\prime} \int_{-\infty}^{t^{\prime}} d t^{\prime \prime} \int d^{3} k \\
& \quad \times V_{\mathbf{p}_{2} e}^{(c)} V_{\mathbf{p}_{1} e, \mathbf{k} g}^{(c)} V_{\mathbf{k} g}^{(c)} \exp \left[i S^{(c)}\left(\mathbf{p}_{1}, \mathbf{p}_{2}, \mathbf{k}, t, t^{\prime}, t^{\prime \prime}\right)\right],
\end{aligned}
$$

where the action

$$
\begin{aligned}
& S^{(c)}\left(\mathbf{p}_{1}, \mathbf{p}_{2}, \mathbf{k}, t, t^{\prime}, t^{\prime \prime}\right) \\
& =E_{1 g} t^{\prime \prime}+E_{2 g} t^{\prime}+E_{2 e}^{(c)}\left(t-t^{\prime}\right)-\int_{t^{\prime \prime}}^{t^{\prime}} \frac{[\mathbf{k}+\mathbf{A}(\tau)]^{2}}{2} d \tau \\
& \quad-\int_{t^{\prime}}^{\infty} \frac{\left[\mathbf{p}_{1}+\mathbf{A}(\tau)\right]^{2}}{2} d \tau-\int_{t}^{\infty} \frac{\left[\mathbf{p}_{2}+\mathbf{A}(\tau)\right]^{2}}{2} d \tau
\end{aligned}
$$

describes the process in which an electron, initially at a bound state of energy $-E_{1 g}$, leaves at $t^{\prime \prime}$, returns to the core at $t^{\prime}$ with the intermediate momentum $\mathbf{k}$, and excites a second electron from a state with energy $-E_{2 g}$ to a state with energy $-E_{2 e}^{(c)}$. The first electron is released at $t^{\prime}$ with momentum $\mathbf{p}_{1}$, while the second electron is freed at a subsequent time $t$ with a final momentum $\mathbf{p}_{2}$. The prefactors $V_{\mathbf{k} g}^{(c)}, V_{\boldsymbol{p}_{1} e, \mathbf{k} g}^{(c)}$, and $V_{\boldsymbol{p}_{2} e}^{(c)}$ are related to the ionization of the first electron, the recollision-excitation process and the tunnel ionization of the second electron, respectively. They contain all information about the interactions, which are chosen as long range, and about the electronic bound states $[14,15]$. We compute the transition amplitude (1) using the steepest descent method, as described in Ref. [21]. We use the notation $p_{n \|}$ and $\mathbf{p}_{n \perp}, n=1,2$, for the momentum components parallel and perpendicular to the laser-field polarization, respectively. We employ a monochromatic field, which is a good approximation for longer pulses [22] and is reasonable for short pulses if the carrier-envelope phase (CEP) is averaged out. For a resolved CEP, more care must be taken (see our previous work [5,22-24] for details). However, this is not the scenario addressed in this Letter.

Because of momentum the constraints attributed to the rescattering of the first electron and the ionization of the second electron occurring, most probably, near field crossings and crests, respectively, the distributions will be located around $\left(p_{1 \|}, p_{2 \|}\right)=\left( \pm 2 \sqrt{U_{p}}, 0\right)$. Considering the symmetry of displacement by half a cycle and the particle exchange of the system leads to four "events" located around $\left(-2 \sqrt{U_{p}}, 0\right),\left(2 \sqrt{U_{p}}, 0\right),\left(0,2 \sqrt{U_{p}}\right)$, and $\left(0,-2 \sqrt{U_{p}}\right)$. We label these event amplitudes as $M_{l}^{(c)}$, $M_{u}^{(c)}, M_{r}^{(c)}$, and $M_{d}^{(c)}$, given that they occupy the left, upper, right, and lower regions of the parallel momentum plane, respectively. Quantum interference of events occurs mainly in the overlap of the above regions, which is located around the diagonals $p_{1 \|}= \pm p_{2 \|}$. For a single channel, the coherent sum of such amplitudes, integrated over the transverse-momentum components, reads

$$
\Omega^{(c)}\left(p_{1 \|}, p_{2 \|}\right)=\iint d^{2} p_{1 \perp} d^{2} p_{2 \perp}\left|M^{(c)}\right|^{2},
$$

with $M^{(c)}=\sum_{\nu} M_{\nu}^{(c)}$, and $\nu=l, r, u, d$. If the events are summed incoherently, the amplitudes are replaced by probabilities; i.e., $\sum_{\nu}\left|M_{\nu}^{(c)}\right|^{2}$ is employed instead. The corresponding expression for more than one channel is

$$
\Omega\left(p_{1 \|}, p_{2 \|}\right)=\iint d^{2} p_{1 \perp} d^{2} p_{2 \perp}\left|\sum_{c} M^{(c)}\right|^{2} .
$$

Equation (4) assumes that each excitation represents a path, which the second electron can take. Hence, the amplitudes corresponding to each path must be summed. One may include different amplitudes or phases for each channel, which would model channel selection or account for phase effects. This yields a more general sum $\int d^{2} p_{1 \perp} d^{2} p_{2 \perp}\left|\sum_{c} N_{c} e^{i \phi_{c}} M^{(c)}\right|^{2}$. In Fig. 1, we provide a schematic representation of the event and interchannel interference for the excitation channels in Table I.

Experimental results from Ref. [12] of RESI in argon show that increasing pulse lengths from few to many cycle pulses causes a transition from cross-shaped to slightly anticorrelated, correlated or ring-shaped distributions. Our results, displayed in Fig. 2, exhibit many of these features. Specifically, we can model the transition from a crossshaped to an anticorrelated distribution, as shown in the first three rows of Fig. 2. In Figs. 2(e)-2(1), we present focal-averaged RESI distributions for the same parameters as in the upper panels of Fig. 2, and similar focusing conditions as in Refs. [12,25]. In the latter case, the coherent sums of events and channels are very similar to those obtained for a fixed peak intensity, with some enhancement along the antidiagonal for specific parameters [see Figs. 2(f) and 2(g)]. Moreover, we can see in Figs. 2(m) and 2(n) that the distributions go from anticorrelated to correlated for an increasing laser-field intensity.

These effects are achieved by using coherent superpositions of the excitation channels given in Table I, using the
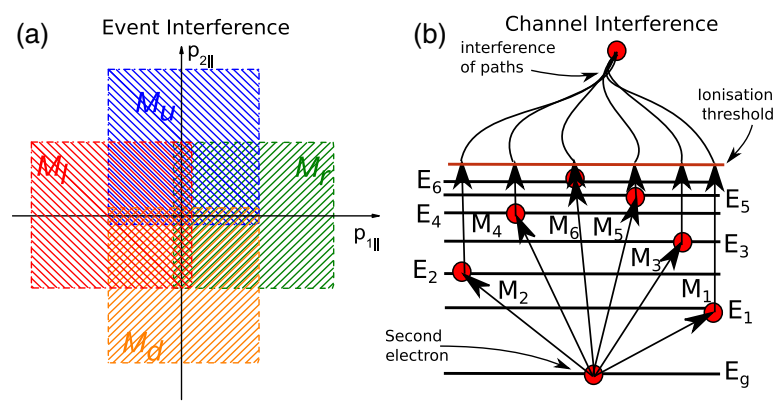

FIG. 1. Schematic representation of the event and interchannel interference for RESI [(a) and (b), respectively]. The shaded regions in (a) represent regions where $M_{l}^{(c)}, M_{r}^{(c)}, M_{u}^{(c)}$, and $M_{d}^{(c)}$ are substantial, and the arrows in (b) indicate the different excitation pathways for the second electron, according to Table I. 
TABLE I. Excitation channels for $\mathrm{Ar}^{+}$employed in this Letter. From left to right, the columns give the number associated with the channel, the electronic configurations for the sublevels involved in the excitation, and the absolute value $E_{2 e}$ of the excited-state energy, respectively. The excitation pathway for the second electron is given in parentheses.

\begin{tabular}{lcc}
\hline \hline Channel & Transition & $E_{2 e}$ (a.u.) \\
\hline 1 & $3 s 3 p^{6}(3 s \rightarrow 3 p)$ & 0.52 \\
2 & $3 p^{5} 3 d(3 p \rightarrow 3 d)$ & 0.41 \\
3 & $3 p^{5} 4 s(3 p \rightarrow 4 s)$ & 0.4 \\
4 & $3 p^{5} 4 p(3 p \rightarrow 4 p)$ & 0.31 \\
5 & $3 p^{5} 4 d(3 p \rightarrow 4 d)$ & 0.18 \\
6 & $3 p^{5} 5 s(3 p \rightarrow 5 s)$ & 0.19 \\
\hline \hline
\end{tabular}

relative weights and phases in Table II. The phases have been chosen only once, and we sought to achieve an anticorrelated pattern in Fig. 2(c). This panel could be easily matched to the experimental counterpart in Ref. [12] for a pulse long enough so that a monochromatic field was a good approximation. For

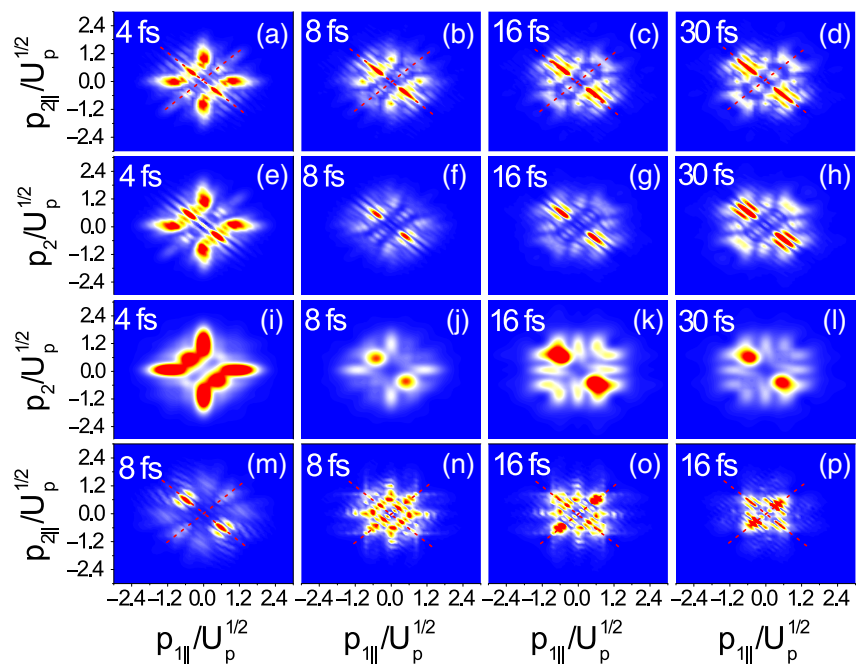

FIG. 2. RESI distributions for argon $\left(E_{1 g}=0.58\right.$, $E_{2 g}=1.02$ a.u.), computed using different coherent superpositions of the excitation channels in Table I. The phases and weights employed in these superpositions are provided in Table II, and they have been chosen in such a way as to reproduce the experimental data from Ref. [12]. In the first row, (a)-(d), the peak intensity is $1.0 \times 10^{14} \mathrm{~W} / \mathrm{cm}^{2}$, with $\omega=0.057$ a.u. and a ponderomotive energy of $U_{p}=0.22 \mathrm{a}$.u. The second row is the same as the first except that the focal averaging has been used. The third row is an incoherent sum of events and uses focal averaging. To incorporate focal averaging we integrate the expression from 0 to the peak intensity as in Eq. (7) in the Supplemental Material [25]. The peak intensities in the fourth row are as follows: (m) $I=0.8 \times 10^{14} \mathrm{~W} / \mathrm{cm}^{2}$ with $U_{p}=0.18$ a.u., (n) and (o) $I=1.2 \times 10^{14} \mathrm{~W} / \mathrm{cm}^{2}$ with $U_{p}=0.26$ a.u., and (p) $I=1.4 \times 10^{14} \mathrm{~W} / \mathrm{cm}^{2}$ with $U_{p}=$ 0.30 a.u. and where no focal averaging has been used. The dashed lines in the figure indicate the diagonals $p_{1 \|}= \pm p_{2 \|}$. The numbers on the top left corner of the panels indicate the pulse length in Ref. [12] that we are aiming to reproduce.
TABLE II. Coherent superpositions employed in Fig. 2. The letters in the first column indicate the panels in Fig. 2 for which a specific superposition has been employed. From the second to the seventh column, the numbers in the first row indicate the excitation channel in Table I, and the excited state of the second electron is given in parentheses. The numbers $N_{c} e^{i \phi_{c}}$ give the weight and the relative phase for each channel.

\begin{tabular}{lcccccc}
\hline Panel (Fig. 2) & $1(3 p)$ & $2(3 d)$ & $3(4 s)$ & $4(4 p)$ & $5(4 d)$ & $6(5 s)$ \\
\hline (a) & $1 e^{i \pi / 4}$ & $1 e^{0}$ & $4 e^{i 7 \pi / 8}$ & $1 e^{i 7 \pi / 8}$ & $0.5 e^{i \pi / 2}$ & $4 e^{i \pi / 2}$ \\
(b), (e), (f) & $1 e^{i \pi / 4}$ & $1 e^{0}$ & $2 e^{i 7 \pi / 8}$ & $1 e^{i 7 \pi / 8}$ & $0.6 e^{i \pi / 2}$ & $2 e^{i \pi / 2}$ \\
(c), (g), (h) & $1 e^{i \pi / 4}$ & $1 e^{0}$ & $1 e^{i 7 \pi / 8}$ & $1 e^{i 7 \pi / 8}$ & $0.7 e^{i \pi / 2}$ & $1 e^{i \pi / 2}$ \\
(d) & $1 e^{i \pi / 4}$ & $1 e^{0}$ & $0.5 e^{i 7 \pi / 8}$ & $2 e^{i 7 \pi / 8}$ & $0.8 e^{i \pi / 2}$ & $0.5 e^{i \pi / 2}$ \\
\hline
\end{tabular}

a more detailed discussion of pulse-length effects, see the Supplemental Material [27], Secs. II and III.

If a pattern is optimized to be anticorrelated at one drivingfield strength, changing this will cause it to flip to be correlated. Hence, in Figs. 2(n)-2(p) we obtain correlated patterns, in agreement with Refs. [12,25]. One should note that, despite this myriad of shapes, all intensities employed in this experiment are well within the below-threshold regime, as the maximal kinetic energy of the returning electron, $3.17 U_{p}$, is significantly lower than the second ionization potential $E_{2 g}$ [28]. Hence, RESI is the prevalent NSDI mechanism. Similar superpositions may be used to reproduce the results in Refs. [7,9,10]. In Sec. IV of the Supplemental Material [29], for comparison, we provide examples of RESI distributions employing Eq. (4) without extra phases or amplitude biases.

The features observed in Figs. 2(a)-2(d) mark a change from a regime in which excitation to $s$ states is prevalent to a scenario in which a coherent superposition of $p$ and $d$ states dominates. This statement can be inferred from Fig. 3, which shows very different shapes for different channels of excitation. These differences stem from the prefactor $V_{\mathbf{p}_{2} e}$ related to the ionization of the second electron. For $p$ and $d$ states, $V_{\mathbf{p}_{2} e}$ has angular nodes, which prevent the distributions from being located along the axes. In contrast, for $s$ states, only radial nodes are present, so that the electron-momentum distributions will be cross shaped [14,30]. None of the coherent distributions in Figs. 2 and 3 exhibit the fourfold symmetry obtained if incoherent sums are used. This symmetry breaking occurs already for a single channel, with fringes along and parallel to the two diagonals. These fringes may be understood if one considers a single-channel coherent sum equation (3) and its incoherent counterpart, neglecting the prefactors (see Fig. 4). They come from the interference of the amplitudes associated with electron indistinguishability. The diagonal maxima and minima satisfy the condition $p_{1 \|}=p_{2 \|} \pm|\delta|$, with $|\delta| \simeq \omega n \pi /$ $\left(2 \sqrt{U_{p}}\right)$, where $n$ is even or odd, respectively. The antidiagonal fringes are four times narrower. The interevent fringes are obtained analytically, and they have been derived elsewhere [30]. Remarkably, all interference effects also survive focal averaging. This is shown in Figs. 2(e)-2(h), 


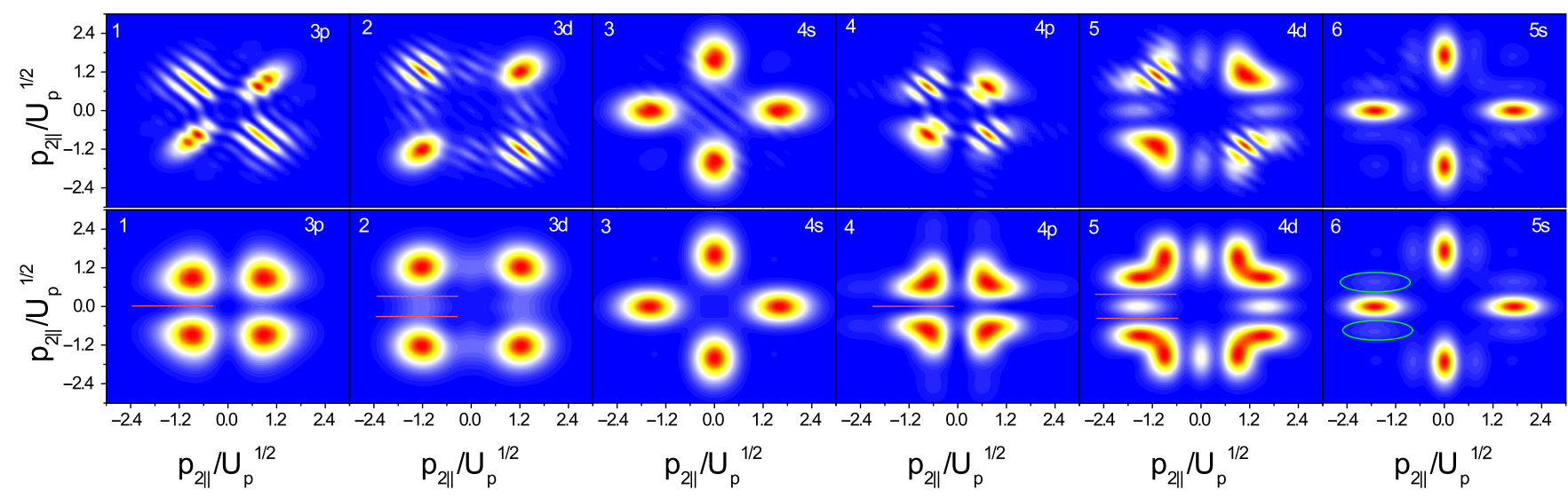

FIG. 3. One-channel RESI electron-momentum distributions computed for argon, with all prefactors included, for the parameters in Table I. The laser intensity and frequency are $I=4.56 \times 10^{13} \mathrm{~W} / \mathrm{cm}^{2}$ and $\omega=0.057$ a.u., respectively. Each panel has been labeled with the corresponding channel number (top left), as stated in Table I, and the excited state of the second electron (top right). The top and bottom rows display a coherent and an incoherent sum of events, respectively. Angular and radial nodes from $V_{\mathbf{p}_{2} e}$ have been marked on the bottom row with red lines and green circles, respectively.

where both types of interference are visible. For incoherent event superpositions, the hyperbolic interchannel fringes become quite evident [Figs. 2(j)-2(1)].

In summary, we have shown that two types of quantum interference, which survive transverse-momentum integration and focal averaging, play an important role in reproducing results similar to those in experiments. Event interference will break the fourfold symmetry previously seen in the SFA, even when only considering a single channel of excitation. Interchannel interference can be used to maximize anticorrelation and correlation and alter the shape of the electron-momentum distributions, and to thus create all distributions found experimentally [7,9,10,12]. This brings additional insight into many experimental studies $[7,9,12]$, in which correlated distributions found for increasing driving-field intensity have been attributed to direct ionization, despite still being below the threshold intensity [10]. Our results suggest that this could in fact be RESI, provided an appropriate coherent superposition of channels is chosen. Furthermore, recent experiments have shown by using a two-color field and changing the relative phase of the colors, the momentum distributions for NSDI below the electron-impact threshold can be manipulated
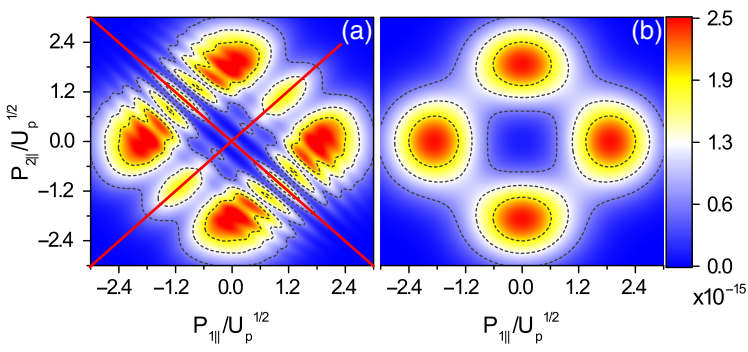

FIG. 4. One-channel coherent and incoherent sums $\Omega^{(c)}$ of all events, computed neglecting the prefactors for the same field parameters as in the previous figure [Figs. 2(a) and 2(b), respectively], and the RESI channel 1 in Table I. The diagonals are indicated with the red lines in the figure. from being anticorrelated to correlated [31]. The phases of each channel and event depend strongly on the field, as they derive from the action. Hence, it would not be unreasonable to assume that this effect came from quantum interference.

The present work, however, invites two main questions: (i) What justifies the use of additional phases and amplitudes? (ii) Why do very short pulses favor excitation to $s$ states, while longer pulses favor $p$ or $d$ channels? The phases and amplitudes in Table II make up for several features which are absent in the present model. First, the residual Coulomb potential modifies the action, leading to phase corrections, and also the amplitudes associated with ionization from specific bound states [32]. This issue has been investigated for a single channel in above-threshold ionization, using Coulomb-corrected forms of the SFA. There is evidence that it significantly changes interference patterns and the topology of the electron orbits in the continuum [39-43]. Second, bound-state depletion and Stark shifts in the bound-state energies enhance ionization from the weakly bound states and modify the times for which the electrons reach the continuum. Third, a short pulse has a certain width in frequency and intensity. Fourth, there is some experimental uncertainty in the determination of the peak-field intensity. Fifth, the core dynamics may be important [48]. All of this will change the phase differences between channels and events and the dominant channels. The prevalence of $s$-state contributions for short pulses is related to how the relative contribution of each channel varies with the field frequency and intensity. The $s$-state contributions dominate for low intensities and frequencies. For the parameters in this Letter, this regime can only be reached if there is a large frequency spread and a variation in the peak intensity, i.e., for short pulses. This is addressed in more detail in Sec. III of the Supplemental Material [49].

Finally, given the important role quantum interference has in RESI, one should consider the implications that this has for classical models. We have shown that interference 
hugely increases the SFA's descriptive power and can reproduce many of the features seen in the experiments. These features are obtained without the need to resort to multiple collisions, and they are related to the geometry of specific intermediate bound-state superpositions [50]. Thus, classical trajectories should be used with caution. Nonetheless, a large density of states may lead to a quasicontinuum, which could give rise to quasiclassical wave packets where interference would play less of a role. Our implementation of the SFA currently neglects broadening of states caused by the field. However, recent studies of the RESI phase-space dynamics have revealed a highly confined region that can be associated with trapping in an excited state [53]. This would justify using discrete bound states and would render interference important. This provides a large scope for RESI to be used as an attosecond-imaging tool in order to probe and reconstruct the intermediate state of the second electron.

We thank J. Chen and X. Liu for the useful discussions, B. Bergues for calling [12] to our attention and information on focal averaging, J. Dumont for his help with his complex Bessel function library [54] and the UK EPSRC (Grant No. EP/J019240/1) for the financial support. The authors acknowledge the use of the UCL Legion High-Performance Computing Facility (Legion@UCL), and associated support services, in the completion of this work.

*andrew.maxwell.14@ucl.ac.uk

†c.faria@ucl.ac.uk

[1] P. B. Corkum, Phys. Rev. Lett. 71, 1994 (1993).

[2] C. Figueira de Morisson Faria and X. Liu, J. Mod. Opt. 58, 1076 (2011); W. Becker, X. Liu, P. J. Ho, and J. H. Eberly, Rev. Mod. Phys. 84, 1011 (2012).

[3] R. Panfili, J. H. Eberly, and S. L. Haan, Opt. Express 8, 431 (2001).

[4] C. Figueira de Morisson Faria, H. Schomerus, X. Liu, and W. Becker, Phys. Rev. A 69, 043405 (2004).

[5] C. Figueira de Morisson Faria, X. Liu, A. Sanpera, and M. Lewenstein, Phys. Rev. A 70, 043406 (2004).

[6] X. Jia, X. Hao, D. Fan, W. Li, and J. Chen, Phys. Rev. A 88, 033402 (2013).

[7] E. Eremina et al., J. Phys. B 36, 3269 (2003).

[8] D. Zeidler, A. Staudte, A. B. Bardon, D. M. Villeneuve, R. Dörner, and P. B. Corkum, Phys. Rev. Lett. 95, 203003 (2005).

[9] Y. Liu, S. Tschuch, A. Rudenko, M. Dürr, M. Siegel, U. Morgner, R. Moshammer, and J. Ullrich, Phys. Rev. Lett. 101, 053001 (2008).

[10] Y. Liu, D. Ye, J. Liu, A. Rudenko, S. Tschuch, M. Dürr, M. Siegel, U. Morgner, Q. Gong, R. Moshammer, and J. Ullrich, Phys. Rev. Lett. 104, 173002 (2010).

[11] B. Bergues et al., Nat. Commun. 3, 813 (2012).

[12] M. Kübel et al., New J. Phys. 16, 033008 (2014).

[13] X. Sun, M. Li, D. Ye, G. Xin, L. Fu, X. Xie, Y. Deng, C. Wu, J. Liu, Q. Gong, and Y. Liu, Phys. Rev. Lett. 113, 103001 (2014).
[14] T. Shaaran, M. T. Nygren, and C. Figueira de Morisson Faria, Phys. Rev. A 81, 063413 (2010).

[15] T. Shaaran and C. Figueira de Morisson Faria, J. Mod. Opt. 57, 984 (2010).

[16] Z. Chen, Y. Liang, and C. D. Lin, Phys. Rev. A 82, 063417 (2010)

[17] A. Emmanouilidou and A. Staudte, Phys. Rev. A 80, 053415 (2009).

[18] D. F. Ye and J. Liu, Phys. Rev. A 81, 043402 (2010).

[19] L. Zhang et al., Phys. Rev. A 90, 061401(R) (2014).

[20] X. Hao, J. Chen, W. Li, B. Wang, X. Wang, and W. Becker, Phys. Rev. Lett. 112, 073002 (2014).

[21] C. Figueira de Morisson Faria, H. Schomerus, and W. Becker, Phys. Rev. A 66, 043413 (2002).

[22] X. Liu and C. Figueira de Morisson Faria, Phys. Rev. Lett. 92, 133006 (2004).

[23] T. Shaaran, C. Figueira de Morisson Faria, and H. Schomerus, Phys. Rev. A 85, 023423 (2012).

[24] C. Figueira de Morisson Faria, T. Shaaran, and M. T. Nygren, Phys. Rev. A 86, 053405 (2012).

[25] See Supplemental Material at http://link.aps.org/ supplemental/10.1103/PhysRevLett.116.143001, which includes Refs. [26], for details on how the focal averaging employed in this Letter was performed.

[26] R. Kopold, W. Becker, M. Kleber, and G. G. Paulus, J. Phys. B 35, 217 (2002).

[27] See Supplemental Material at http://link.aps.org/ supplemental/10.1103/PhysRevLett.116.143001, for details on pulse-length effects.

[28] For the intensities in Fig. 2, this energy varies from 0.55 to 0.86 a.u., i.e., between $54 \%$ and $84 \%$ of $E_{2 g}$.

[29] See Supplemental Material at http://link.aps.org/ supplemental/10.1103/PhysRevLett.116.143001, for RESI distributions without additional phases and normalizations.

[30] A. S. Maxwell and C. Figueira de Morisson Faria, Phys. Rev. A 92, 023421 (2015).

[31] L. Zhang et al., Phys. Rev. Lett. 112, 193002 (2014).

[32] See Supplemental Material at http://link.aps.org/ supplemental/10.1103/PhysRevLett.116.143001, which includes Ref. [33-38], for details on Coulomb-corrected approaches.

[33] O. Smirnova, M. Spanner, and M. Ivanov, J. Phys. B 39, S307 (2006); 39, S323 (2006).

[34] O. Smirnova, M. Spanner, and M. Ivanov, Phys. Rev. A 77, 033407 (2008).

[35] S. V. Popruzhenko and D. Bauer, J. Mod. Opt. 55, 2573 (2008).

[36] S. V. Popruzhenko, G. G. Paulus, and D. Bauer, Phys. Rev. A 77, 053409 (2008).

[37] S. V. Popruzhenko, J. Phys. B 47, 204001 (2014).

[38] L. Torlina, M. Ivanov, Z. B. Walters, and O. Smirnova, Phys. Rev. A 86, 043409 (2012).

[39] T. M. Yan, S. V. Popruzhenko, M. J. J. Vrakking, and D. Bauer, Phys. Rev. Lett. 105, 253002 (2010).

[40] T. M. Yan and D. Bauer, Phys. Rev. A 86, 053403 (2012).

[41] L. Torlina, J. Kaushal, and O. Smirnova, Phys. Rev. A 88, 053403 (2013).

[42] X. Y. Lai, C. Poli, H. Schomerus, and C. Figueira de Morisson Faria, Phys. Rev. A 92, 043407 (2015).

[43] See Supplemental Material at http://link.aps.org/ supplemental/10.1103/PhysRevLett.116.143001, which 
includes Refs. [44-47], for interference effects which can only be explained by resorting to the interplay between the Coulomb potential and the laser field.

[44] A. Rudenko, K. Zrost, C. D. Schröter, V. L. B. de Jesus, B. Feuerstein, R. Moshammer, and J. Ullrich, J. Phys. B 37, L407 (2004).

[45] Z. J. Chen, T. Morishita, A.-T. Le, M. Wickenhauser, X. M. Tong, and C. D. Lin, Phys. Rev. A 74, 053405 (2006).

[46] D. G. Arbó, S. Yoshida, E. Persson, K. I. Dimitriou, and J. Burgdörfer, Phys. Rev. Lett. 96, 143003 (2006).

[47] D. G. Arbó, K. I. Dimitriou, E. Persson, and J. Burgdörfer, Phys. Rev. A 78, 013406 (2008).

[48] O. Smirnova, Y. Mairesse, S. Patchkovskii, N. Dudovich, D. Villeneuve, P. Corkum, and M. Yu. Ivanov, Nature (London) 460, 972 (2009).

[49] See Supplemental Material at http://link.aps.org/ supplemental/10.1103/PhysRevLett.116.143001, for addi- tional information on how the dominant channels change with the pulse length.

[50] Within classical models, it has been argued that anticorrelated distributions result from multiple recollisions, which provide the electrons with enough energy so that they can overcome the Stark saddle and reach the continuum. In Refs. [12,51], this has been related to the prevalence of anticorrelation for longer pulses. Nonetheless, multiple recollisions may not be the only classical mechanism causing anticorrelation, as classical-trajectory computations obtained anticorrelated RESI distributions for much shorter times [17,52].

[51] C. Huang, Y. Zhou, Q. Zhang, and P. Lu, Opt. Express 21, 11382 (2013).

[52] B. Yu, D. Zhang, Y. Li, and Q. Tang, J. Mod. Opt. 59, 679 (2012).

[53] F. Mauger, A. Kamor, C. Chandre, and T. Uzer, Phys. Rev. Lett. 108, 063001 (2012).

[54] See https://github.com/valandil/complex_bessel. 